\title{
Valor pronóstico de la última clasificación histológica de tumores mamarios caninos
}

\author{
Rosciani, A.S.; Merlo, W.A.; Insfrán, R.M.; Rodríguez, Y.N. \\ Servicio de Diagnóstico Histopatológico y Citológico, Facultad de Ciencias Veterinarias, Universidad \\ Nacional del Nordeste, Sargento Cabral 2139, Corrientes (3400), Argentina. Tel: 0379-4425753 (int. 192), \\ E-mail: arosciani@vet.unne.edu.ar
}

\begin{abstract}
Resumen
Rosciani, A.S.; Merlo, W.A.; Insfrán, R.M.; Rodríguez, Y.N.: Valor pronóstico de la última clasificación histológica de tumores mamarios caninos. Rev. vet. 26: 2, 99-102, 2015. El presente trabajo tuvo como objetivo determinar el valor pronóstico de la aplicación de la clasificación histológica avalada por la Asociación Mundial de Veterinaria de Pequeños Animales para los tumores mamarios caninos, al relacionarla con el grado histológico de malignidad de cada tumor y la sobrevida de las pacientes. Se trabajó con tumores mamarios de 50 hembras caninas cuya evolución post-mastectomía fue objeto de seguimiento durante 18 meses para determinar su sobrevida, por medio de curvas de Kaplan-Meier. Todos los casos estudiados fueron carcinomas de las siguientes variedades: simple tubular (4), simple túbulopapilar (8), micropapilar invasor (1), anaplásico (1), de tipo complejo (17), de tipo mixto (15) y carcinoma y mioepitelioma maligno (4). La graduación histológica de malignidad mostró la siguiente distribución: grado I (19), grado II (29) y grado III (2). Los resultados hallados no permiten aún correlacionar cada variedad de esta clasificación histológica con un pronóstico determinado.
\end{abstract}

Palabras clave: canino, neoplasias mamarias, diagnóstico histopatológico, graduación de malignidad.

\begin{abstract}
Rosciani, A.S.; Merlo, W.A.; Insfrán, R.M.; Rodríguez, Y.N.: Predictive value of the last canine mammary tumours histologic classification. Rev. vet. 26: 2, 99-102, 2015. This classification, proposed by the American College of Veterinary Pathologist's Oncology Comittee and Word Small Animal Veterinary Association, was assessed by comparing the malignancy grade of each tumor and patient's survival. Fifty bitches with spontaneous mammary neoplasms were clinically followed during 18 month after mastectomy to determine Kaplan-Meier survival curves. The studied neoplasms were diagnosed as carcinomas of the following varieties: tubular (4), tubulopapillary (8), micropapillary invasive (1), anaplastic (1), complex type (17), mixed type (15) and carcinoma and malignant myoepithelioma (4). Histological graduation showed 19 cases of grade I, 29 cases of grade II, with only 2 cases diagnosed as grade III. Considering the results, a complete correlation between each variety of this classification and a specific evolution is not possible with current information.
\end{abstract}

Key words: dog, mammary neoplasms, histopathological diagnosis, predictive value.

\section{INTRODUCCIÓN}

En medicina veterinaria los tumores de glándula mamaria son clasificados de acuerdo con el tipo histopatológico, así como con el grado histológico de malignidad, tamaño del tumor, índice de proliferación, estado de los nódulos linfáticos, expresión de los receptores

Recibido: 10 junio 2015 / Aceptado: 22 septiembre 2015 Presentado Reunión Comunic.Científ. FCV-UNNE 2014. Proyecto SGCYT-UNNE - B016/2010 hormonales y de otras moléculas, en búsqueda de herramientas que actúen como indicadores de pronóstico ${ }^{2}$.

El "estándar de oro" continúa siendo el diagnóstico histopatológico, debido a que permite conocer datos referidos a la extensión local de la neoplasia, invasión vascular, presencia de necrosis y otros. La descripción de éstas y otras características histológicas tienen, en mayor o menor medida, valor pronóstico.

Las neoplasias mamarias caninas se caracterizan por su heterogeneidad histológica, por lo cual las clasificaciones utilizadas sufren constantes revisiones. La 
Organización Mundial de la Salud ha venido proponiendo revisiones sucesivas, como las de los años 1974 y $1999^{5}$. La clasificación vigente, propuesta por el American College of Veterinary Pathologist's Oncology Comittee y avalada por la Word Small Animal Veterinary Association para neoplasias mamarias caninas, es la elaborada en 2011 por Goldschmidt et al. ${ }^{3}$.

Tal clasificación incluye cinco nuevos subtipos morfológicos de carcinomas mamarios caninos en comparación con la clasificación de la OMS de $1999^{1}$ : el carcinoma micropapilar invasor, el comedocarcinoma, el carcinoma ductal, el carcinoma papilar intraductal y el carcinoma y mioepitelioma malignos ${ }^{7}$. El valor pronóstico de esta clasificación se encuentra a prueba y constituye el objetivo de este trabajo.

\section{MATERIAL Y MÉTODOS}

Se trabajó con muestras histopatológicas de tumores mamarios de cincuenta pacientes caninas que concurrieron al Hospital de Clínicas de la Facultad de Ciencias Veterinarias de la Universidad Nacional del Nordeste (Corrientes, Argentina).

Luego de la extirpación quirúrgica de los tumores se realizó la fijación de los mismos en formol bufferado al $10 \%$ para su remisión al Servicio de Diagnóstico Histopatológico y Citológico de dicha Facultad. El procesamiento del material se llevó a cabo siguiendo la técnica histológica clásica. El diagnóstico histopatológico se efectuó en base a la clasificación histológica para las neoplasias mamarias caninas, publicada por Goldschmidt et al. en el año $2011^{3}$.

Además, se determinó el grado de malignidad (GM) histológico de los carcinomas mamarios ${ }^{6}$, que cuantifica tres características: la formación de túbulos, el pleomorfismo nuclear y el índice mitótico, estableciendo tres posibles grupos de malignidad creciente.

Durante dieciocho meses post-mastectomía, las pacientes fueron evaluadas clínicamente y registrada su evolución. Posteriormente, se establecieron comparaciones entre los distintos tipos histológicos (TH) tumorales diagnosticados y su GM, y entre los TH y la evolución de las pacientes (vivas y muertas a dieciocho meses post cirugía), al mismo tiempo que se confeccionaron curvas de supervivencia mediante el programa Infostat/Profesional, versión 10 (Estadística y Diseño, FCA, Universidad Nacional de Córdoba, 2010).

\section{RESULTADOS}

Las neoplasias estudiadas correspondieron a diferentes variedades histológicas de carcinomas mamarios caninos. Los hallazgos se consignan en la Tabla 1.
Tabla 2. Evolución de las pacientes agrupada según el grado de malignidad.

\begin{tabular}{lccc}
\hline malignidad & vivas & muertas & total \\
\hline GM I & 12 & 7 & 19 \\
GM II & 11 & 18 & 29 \\
GM III & 0 & 2 & 2 \\
Total & 23 & 27 & 50 \\
\hline
\end{tabular}

GM: grados de malignidad histológica.

Se encontraron diferencias significativas al comparar la distribución de los grados de malignidad en los distintos tipos histológicos $(\mathrm{p}<0,0001)$, no así al considerar la evolución (vivas o muertas a los 18 meses: $\mathrm{p}=0,7117)$.

En la Tabla 2 se presentan pacientes vivas y muertas según el grado de malignidad encontrado; en esta distribución, tampoco se obtuvieron diferencias significativas $(p=0,0946)$.

Se confeccionaron curvas de sobrevida de KaplanMeier de las pacientes agrupadas según el diagnóstico histológico del tumor y también de acuerdo con su grado de malignidad (Figuras 1 y 2 , respectivamente).

\section{Curvas de sobrevida (Kaplan-Meier)}

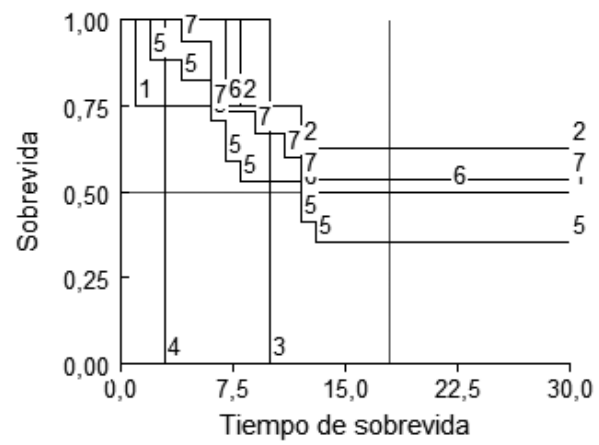

Figura 1. Curvas de Kaplan-Meier según el diagnóstico histológico del tumor. 1: carcinoma simple tubular; 2: carcinoma simple tubulopapilar; 3: carcinoma micropapilar invasor; 4: carcinoma anaplásico; 5: carcinoma complejo; 6: carcinoma y mioepitelioma maligno; 7: carcinoma mixto. 


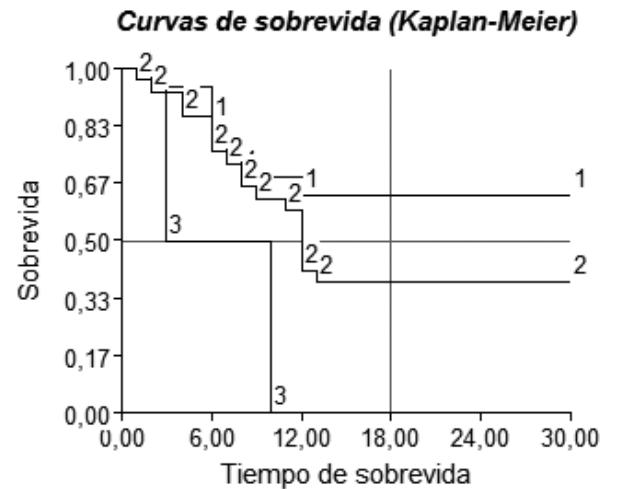

Figura 2. Curvas de Kaplan-Meier según el grado de malignidad tumoral. 1: GM I, 2: GM II., 3: GM III.

La comparación de datos de sobrevida de los distintos tipos histológicos mostró diferencias significativas $(p=0,042)$, pero no ocurrió lo mismo al comparar la sobrevida entre los distintos grados de malignidad $(\mathrm{p}=0,053)$.

\section{DISCUSIÓN}

La aplicación de la clasificación histológica de Goldshmidt et al. (2011) ${ }^{3}$ a los tumores mamarios caninos analizados en este trabajo, evidenció un porcentaje

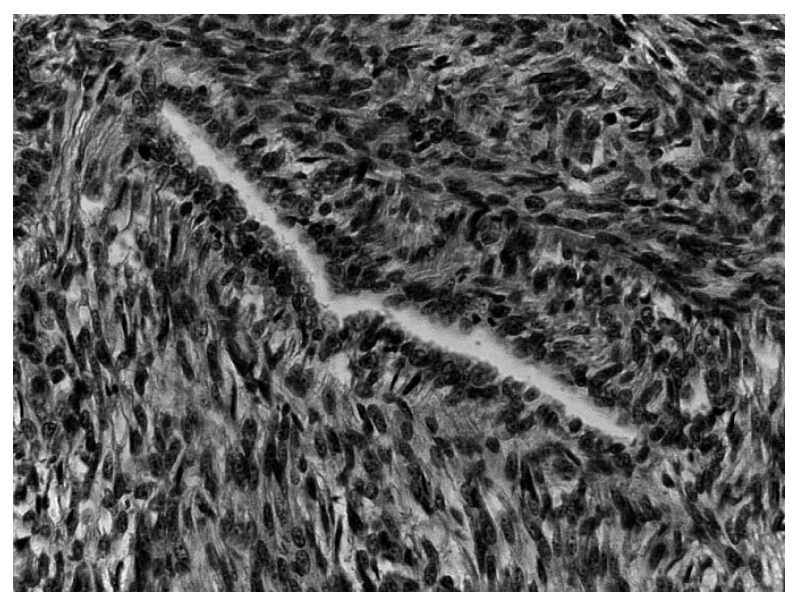

Figura 3. Carcinoma y mioepitelioma maligno (HyE, 400x).

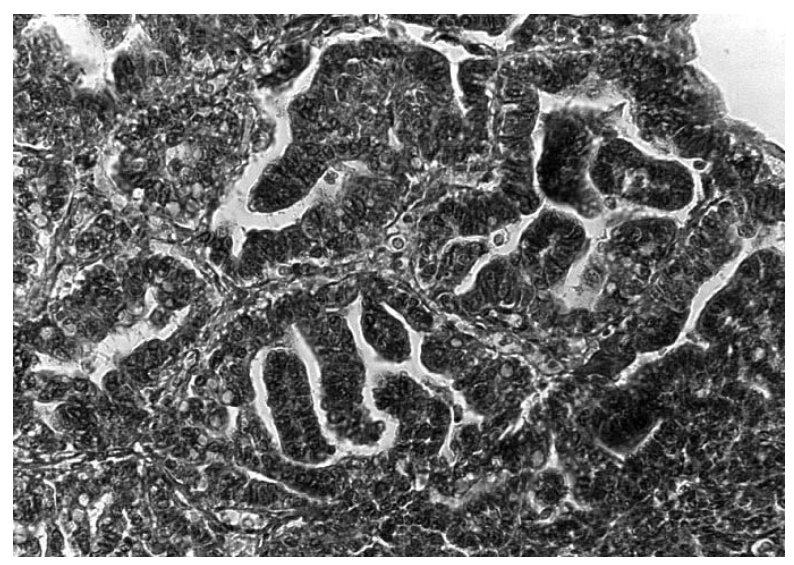

Figura 4. Carcinoma micropapilar invasor (HyE, 100x). mayor de casos diagnosticados como carcinomas de tipo complejo y mixto, que no se corresponde con lo informado por otros investigadores ${ }^{1,2,4}$, quienes mencionan como más frecuentes a los carcinomas simples en sus distintas variedades.

Además, se han clasificado neoplasias como carcinoma y mioepitelioma maligno (Figura 3) y como carcinoma micropapilar invasor (Figura 4), los cuales corresponden a nuevos tipos histopatológicos que aparecen en esta clasificación y resultan más apropiados que las variedades contempladas en clasificaciones anteriores.

Se observaron diferencias significativas en la distribución del grado de malignidad en los diferentes tipos histológicos tumorales, probablemente debido al escaso número de pacientes correspondientes al GM III. En nuestra casuística los carcinomas simples correspondieron a GM I y GM II, mientras que otros ${ }^{4}$, aunque basados en otra clasificación, informan que un $50 \%$ de los carcinomas simples resultan de GM III.

Las curvas de sobrevida mostraron que el $63 \%$ de las pacientes con neoplasias diagnosticadas como carcinoma simple túbulo-papilar sobrevivió a los 18 meses post-cirugía y también el $53 \%$ de las que presentaron carcinomas de tipo mixto. El $50 \%$ de los casos diagnosticados como carcinomas simples tubulares y los carcinomas y mioepiteliomas malignos, sobrevivió a los 18 meses post cirugía, mientras que los casos diagnosticados como carcinoma complejo, sólo lo hicieron en el $35 \%$. Las dos variedades que resultaron más agresivas fueron el carcinoma anaplásico y el carcinoma micropapilar invasor, muriendo las pacientes antes del año de seguimiento y probablemente, al ser casos únicos, son las variedades que han marcado las diferencias significativas.

Por otra parte, se observó que el $50 \%$ de la población con tumores de GM II, no superó el año de sobrevida, mientras que las pacientes con GM I evidenciaron un porcentaje mayor al $50 \%$ con una supervivencia superior a los 18 meses. En estudios anteriores ${ }^{4}$ se registraró una supervivencia superior al año en pacientes con tumores de GM II, mientras que las de GM I sobrevivían en el $100 \%$ de los casos y las de GM III no superaron los 7 meses en el $50 \%$ de los casos.

Probablemente debido a que el número de casos analizado fue bajo, los hallazgos descriptos no permiten aún atribuir a esta clasificación histológica valor predictivo en cuanto a evolución, por lo que se continuará trabajando en esta área.

\section{REFERENCIAS}

1. Caicedo JA, Iregui CA, Cabarcas M, Acosta G. 2012. Estudio comparativo de la frecuencia de tumores mamarios según sexo, edad y tipo histológico en caninos y humanos en los laboratorios de patología anatómica de la Universidad Nacional de Colombia sede Bogotá. Rev Colomb Ciencia Anim 5: 37-46. 
2. Diessler ME. 2009. Carcinomas mamarios de caninos: influencia de variables histológicas e inmunohistoquímicas en el pronóstico. Tesis doctoral, Facultad de Ciencias Veterinarias, Universidad Nacional de La Plata, Argentina, p. 206.

3. Goldschmidt M, Peña L, Rasotto R, Zappulli V. 2011. Classification and grading of canine mammary tumors. Vet Pathol 48: 117, http://vet.sagepub.com/content/48/1/117.

4. Karayannopoulou M, Kaldrymidou E, Constantinidis TC, Dessiris A. 2005. Histological grading and prognosis in dogs with mammary carcinomas: application of a human grading method. J Comp Pathol 133: 246-252.

5. Misdorp W, Else RW, Hellmen E, Lipscomb TP. 1999. Histological classification of mammary tumors of the dog and the cat. Publ. Armed Forces Institute of Pathology in cooperation with the American Registry of Pathology and The World Health Organization, Washington DC, USA, $2^{\text {nd }}$ series, vol VII, p. 58.
6. Misdorp W. 2002. Tumors of the mammary gland. In: Tumors in domestic animals (Meuten DJ Ed), $4^{\text {th }}$ ed., Blackwell Publishing, Iowa, USA, p. 575-606.

7. Peña L, De Andrés PJ, Clemente M, Cuesta P, Pérez MD. 2012. Prognostic value of histological grading in no inflammatory canine mammary carcinomas in a prospective study with two-year follow-up: relationship with clinical and histological characteristic. Vet Pathol 50: 94-105.

\section{SCImago Journal \& Country Rank}

\section{Revista Veterinaria aumentó su índice de impacto}

Noticias de Scimago Research Group (Scimago Journals \& Country Ranks, Scopus-Elsevier) comunican que la publicación de nuestra casa, Revista Veterinaria, aumentó su índice de impacto. El índice SJR mide la influencia científica (impacto) del artículo de una revista, expresando cuán importante es el "artículo promedio" de la publicación en la discusión científica global (sistema Thomson Reuters).

Para nuestra revista, tal indicador había sido de 0,03 entre 2008 y 2011, aumentando a 0,05 en 2012 y a 0,11 en 2013, último período evaluado. El aumento fue del $370 \%$. Asimismo, surge para nuestra publicación un sostenido descenso del indicador que relaciona "citas versus autocitas", demostrando que los autores de los artículos están abandonando la práctica de citar sus propias publicaciones anteriores.

Por último, se advierte que según este portal, nuestra "Revista Veterinaria" continúa siendo la única publicación de esta rama de la ciencia que posee índice de impacto en Argentina. Para el resto del cono sur tal distinción recae en Brasil, Chile, Colombia y Venezuela. No registran índice de impacto las revistas de veterinaria de Bolivia, Paraguay, Uruguay, Perú y Ecuador. En revistas de veterinaria, a nivel mundial el mayor índice de impacto (1,22 puntos) recae en Veterinary Microbiology (Amsterdam, Holanda).

Fuente:

$\mathrm{http}: / /$ www.scimagojr.com/journalrank.php?area $=0 \&$ category $=0 \&$ country $=$ AR\&year $=2012 \&$ order $=$ sjr\&min $=0 \& \min \_$type $=\mathrm{cd}$ Retrieved April 4, 2014. 